\title{
An Improved Method for Muscle Activation Detection During Gait
}

\author{
Lanyi Xu, Andy Adler* \\ School of Information Technology and Engineering \\ University of Ottawa, Ottawa, ON K1N 6N5 \\ lanyixu@site.uottawa.ca, adler@site.uottawa.ca
}

\begin{abstract}
Estimation of on-off timing of human skeletal muscles during movement based on surface electromyography (EMG) is an important issue in sport performance and health care applications. Several methods have been proposed for detecting the on and off timing of the muscle. However, little is known about the reliability and accuracy of these methods, which frequently rely on intuitive and heuristic criteria. Some sophisticated techniques have been proposed, but have a disadvantage of heavy computational load and therefore not suitable for real-time online applications. In this study, an improved method is proposed based on double-threshold method of Bonato et al. (1998). It provides a higher sensitivity for activation detection. In addition, the whitening process of the EMG signal is avoided, significantly reducing the computation time.
\end{abstract}

Index Terms-EMG, gait analysis, signal detection.

\section{INTRODUCTION}

Many clinical applications, such as gait analysis and coordination studies, require accurate detection of when, and for how long, muscles are active. The primary instrument for this measurement is the surface electromyogram (EMG), in which a composite electrical signal is measured of the electrical activity in the nearby muscle tissue. The early methods for detecting muscle activation were based on setting a threshold of EMG envelop amplitude to discriminate the background noise from the signal generated by active muscle[1], [6]. Normally, the threshold level is chosen heuristically. This kind of approach is generally not satisfactory, since measured results depend strongly on the choice of threshold[7]. Moreover, these traditional methods do not allow the user to set independently the detection and false alarm probabilities[2].

More recently, a double-threshold detector has been proposed by Bonato et al. [2] specifically for gait analysis. This method operates on the raw myoelectric signal and, does not require any envelope detection. Its performance is controlled by the values of three parameters, false-alarm probability $\left(P_{f a}\right)$, detection probability $\left(P_{d}\right)$, and time resolution. This method is complex and computationally expensive, requiring a whitening of the signal. It has also been reported to not be very sensitive.

In this paper, we propose an improved method based on the double-threshold method. This algorithm is more sensitive, stable and efficient with decreased computational cost.

Asterisk indicates corresponding author.

\section{Theoretical Considerations}

In this section, we first derive the equations that describe the structure of the detector, and then discuss the strategies for selection of its parameters.

\section{A. Derivation of the Algorithm}

A common model for the surface EMG signal recorded during voluntary dynamic contractions is a zero-mean Gaussian process $s(t) \in \mathcal{N}\left(0, \sigma_{s}\right)$ modulated by the muscle activity and corrupted by an independent zero-mean Gaussian additive noise $n(t) \in \mathcal{N}\left(0, \sigma_{n}\right)$. These signals are sampled with a sampling frequency $f_{s}$ which satisfies Nyquist criterion[2].

We assume that the corrupting noise is stationary and the signal is a nonstationary Gaussian processes that is independent between different trials. Thus the signal and noise can be further expressed as $s(\xi, t) \in \mathcal{N}\left(0, \sigma_{s}(t), \xi\right)$ and $n(\xi, t) \in \mathcal{N}\left(0, \sigma_{n}, \xi\right)$, where $\xi$ represents the trial number of the experiment. In our study, it represents the sequence number of foot steps on a treadmill. Given a sampled signal $\left(s_{i}(\xi)\right)$ and noise $\left(n_{i}(\xi)\right)$, the $i$ th measured data value is

$$
x_{i}(\xi)=s_{i}(\xi)+n_{i}(\xi),
$$

where $s_{i}(\xi)$ and $n_{i}(\xi)$ are $s(\xi, i)$ and $n(\xi, i)$, respectively. Given the assumed independent and Gaussian processes, $s(t)$ and $n(t)$, the probability distribution of $x_{i}$ is given as

$$
f\left(x_{i}\right)=\frac{1}{\sqrt{2 \pi\left(\sigma_{s_{i}}^{2}+\sigma_{n}^{2}\right)}} e^{-\frac{x_{i}^{2}}{2\left(\sigma_{s_{i}}^{2}+\sigma_{n}^{2}\right)}} .
$$

We generate an auxiliary sequence, $\left\{z_{i}\right\}$, by summing up all squared measured data values $\left\{x_{i}\right\}$ at the same time instant of different trials, i.e.

$$
z_{i}=\sum_{\xi} x_{i}^{2}(\xi)
$$

where $\xi \in[1, \ldots, \nu], \nu$ is the number of trials. $z_{i}$ has a probability distribution of

$$
\tilde{f}\left(z_{i}\right)=\frac{1}{\sigma_{s_{i}}^{2}+\sigma_{n}^{2}} \chi^{2}\left(z_{i} /\left(\sigma_{s_{i}}^{2}+\sigma_{n}^{2}\right), \nu\right)
$$

where $\chi^{2}(x, \nu)$ represents Chi-square distribution with $\nu$ degrees of freedom. If there is only noise present in the measured data values, e.g., when the muscle is at rest, $z_{i}$ would be given 
by (3) without the signal contribution, and the (3) and (4) become

$$
z_{i}=\sum_{\xi} n_{i}^{2}(\xi)
$$

and

$$
f\left(z_{i}\right)=\frac{1}{\sigma_{n}^{2}} \chi^{2}\left(z_{i} / \sigma_{n}^{2}, \nu\right) .
$$

The probability that a specific noise sample is above a fixed threshold $\zeta$ is

$$
\begin{aligned}
P_{\zeta} & =P[z>\zeta, x(t)=n(t)] \\
& =\int_{\zeta}^{\infty} f\left(z_{i}\right) d z_{i} \\
& =\int_{\frac{\zeta}{\sigma_{n}^{2}}}^{\infty} \chi^{2}(y, \nu) d y .
\end{aligned}
$$

From (7) we can obtain

$$
\zeta=\sigma_{n}^{2} \chi_{-1}^{2}\left(1-P_{\zeta}, \nu\right),
$$

where $\chi_{-1}^{2}(P, \nu)$ is the inverse chi-square cumulative distribution function with $\nu$ degrees of freedom.

Similarly, when signal $s_{i}$ and noise $n_{i}$ are both present, the probability density function is given by (4). It follows that the probability $P_{d k}$ that the $k$ th sample is above the threshold $\zeta$ is given by

$$
\begin{aligned}
P_{d k} & =P\left[z_{i}>\zeta, x_{i}=s_{i}+n_{i}\right] \\
& =\int_{\zeta}^{\infty} \tilde{f}\left(z_{i}\right) d z_{i} \\
& =\int_{\frac{\zeta}{\sigma_{s_{i}}^{2}+\sigma_{n}^{2}}}^{\infty} \chi^{2}(y, \nu) d y \\
& =1-\bar{\gamma}\left(\zeta /\left(\sigma_{s_{i}}^{2}+\sigma_{n}^{2}\right), \nu / 2\right),
\end{aligned}
$$

where $\bar{\gamma}(x, a)$ is incomplete gamma function.

Bonato et. al. use two thresholds, $\zeta$ and $r_{0}$. The second threshold, $r_{0}$, is set up by introducing a detection window, inside which $m$ successive samples are observed. Muscle activation is detected if at least $r_{0}$ out of $m$ successive samples are above $\zeta$. This technique allows more flexibility in the relationship between $P_{f a}$ and probability of detection by increasing the number of parameters that characterize the detector.

\section{B. Selection of Detector Parameters}

Given a user-selected $P_{f a}$, we now calculate the correspond $\zeta$. The probability $P_{r_{0}}\left(r \geq r_{0} ; m\right)$ that at least $r_{0}$ samples out of $m$ exceed the threshold is given by

$$
P_{r_{0}}\left(r \geq r_{0} ; m\right)=\sum_{k=r_{0}}^{m}\left(\begin{array}{c}
m \\
k
\end{array}\right) P^{k}(1-P)^{m-k},
$$

where $P$ is the probability that a single sample crosses the threshold.

Then the false-alarm probability $P_{f a}$ is the probability that noise samples are incorrectly interpreted as signal, and is obtained from (10) by posing $P=P_{\zeta}$, i.e.

$$
P_{f a}=\sum_{k=r_{0}}^{m}\left(\begin{array}{c}
m \\
k
\end{array}\right) P_{\zeta}^{k}\left(1-P_{\zeta}\right)^{m-k}
$$

and similarly the probability of detection, $P_{d}$, is the probability that signal samples, although corrupted by noise, are correctly recognized, and it is obtained by substituting $P$ with $P_{d k}$ in Eq.10, as shown by

$$
P_{d}=\sum_{k=r_{0}}^{m}\left(\begin{array}{c}
m \\
k
\end{array}\right) P_{d k}^{k}\left(1-P_{d k}\right)^{m-k} .
$$

The user sets the desired values $P_{f a}$ and requested time resolution. Tuning the statistical detector consists of choosing the length of the observation window $(m)$ and two thresholds, $r_{0}$ and $\zeta$.

In this study, the sampling rate is $1 \mathrm{kHz}$. In order to achieve a time resolution for $10 \mathrm{~ms}$, the observation window, $m$, must be 10 points. It has been simulated [2] that if $P_{f a}$ is fixed the $P_{d}$ increases as $r_{0}$ decreases. This behavior does not depend on the values assumed by $m$ and SNR and, hence, may be considered as general. It follows that to reach the highest $P_{d}$, the $r_{0}$ should be set to its minimum value of 1 .

After selection of $r_{0}$ and time resolution, the last step is the selection of $\zeta$. Given the user selected $P_{f a}$, Eq. (11) is solved with respect to $P_{\zeta}$. By substituting $P_{\zeta}$ into Eq. (8), we then obtain the first threshold $\zeta$.

\section{EXPERIMENTAL PROCEDURES AND RESUlTS}

\section{A. Measurement of EMG signal}

The three male volunteers (aged 22-30) were required to run on a treadmill and EMG data were acquired from six different muscle groups of the leg. Also, a sensor was put under the heel inside the shoe to provide a synchronization signal for the heel strike. The EMG signals were amplified, with gain of 2,000, and sampled with an A/D card with the synchronizing signal at 1,000 samples per second, after filtering with an appropriate antialiasing filter. Fig. 1 shows representative EMG data from a subject. The raw EMG signals were divided into segments of a single stride (between two consecutive heel strikes) based on the synchronization signal.

The detection algorithm was implemented in Matlab (Mathworks Inc., Natick, MA) on an 1.8 GHz AMD Athlon processor IBM-compatible PC. Software was written to incorporate a Graphical User Interface so that users can monitor the procedures and results visually. Results were calculated for Bonato et. al.'s method and the proposed method using for 9 measurements from 3 subjects for the biceps femoris (BF) and semitendinosus (ST). $P_{f a}$ was set to 0.05 for both method and the results are shown in Figs. 3 and 4.

\section{B. Computation Steps used for Bonato's method}

The steps for Bonatos's detection is as follows:

1) the raw EMG signal, $\left\{x_{i}\right\}$, of one stride is loaded

2) the raw signal is whitened to make the samples of $\left\{x_{i}\right\}$ uncorrelated with each other

3) the squared values of two successive samples of whitening output are summed and the auxiliary sequence, $z_{i}$ is generated using Eq.3

4) a user identifies a segment of background noise using the GUI windows and then the averaged standard deviation of the noise, $\sigma_{n}$, is obtained 


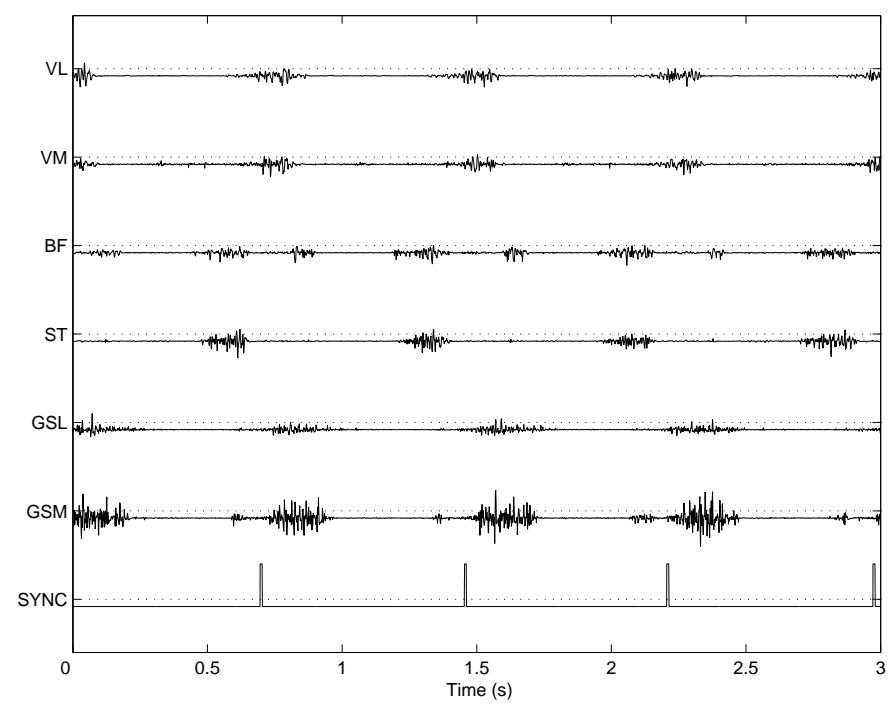

Fig. 1. The raw EMG signals detected from six muscles from a subject on a treadmill. The muscles are the vastus lateralis (VL), the vastus medialis $(\mathrm{VM})$, the biceps femoris (BF), the semi tendinosis (ST), the gastrocnemius lateralis (GSL) and the gastrocnemius medialis (GSM); the last row shows the pressure signal from the heel, which is used for time synchronization

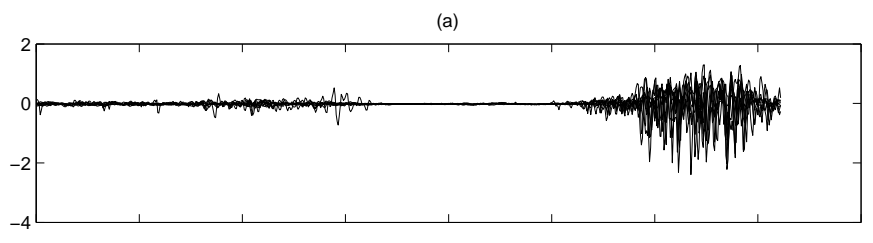

(b)

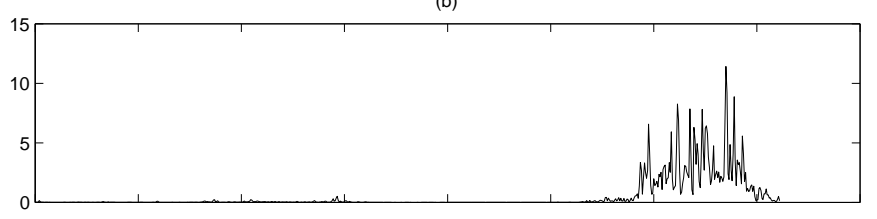

(c)

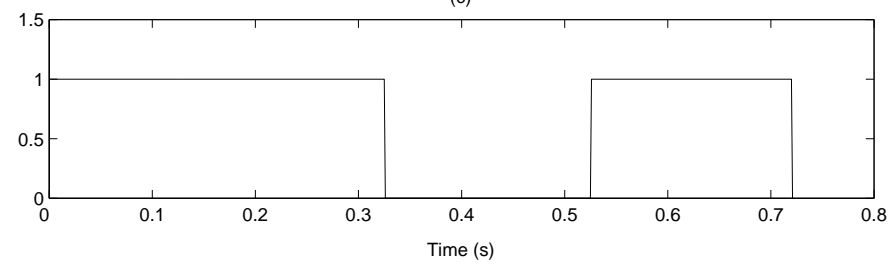

Fig. 2. Representative output from the detection algorithm; (a) the overlay plot of 9 steps raw of EMG signals from Vastus Lateralis muscle, (b) the auxiliary sequence, $z_{i}$, of the raw EMG signal for a strides to be analyzed, (c) the detector output.

5) the width of the detecting window, $m$, is set to 5 , the second threshold, $r_{0}$ is set to 1 and the false-alarm probability, $P_{f a}$, is set to 0.05 and the value of $P_{\zeta}$ calculated for a real root between 0 and 1 in (11)

6) values of $\sigma_{n}$ and $P_{\zeta}$ are substituted into (8) with the $\nu=2$ to calculate $\zeta$

7) the detection window is slid along the auxiliary sequence, $\left\{z_{i}\right\}$. The muscle is considered active, wherever more than $r_{0}$ samples in the detection window have a value greater than $\zeta$

8) the detection sequence obtained in step 7 , is further

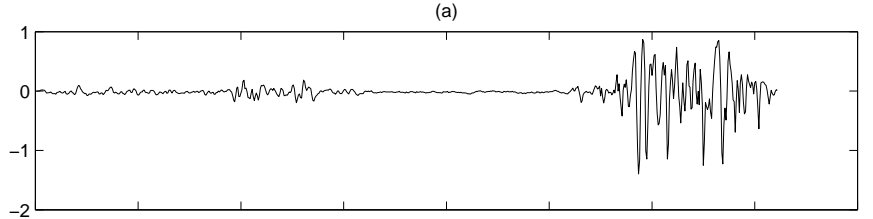

(b)

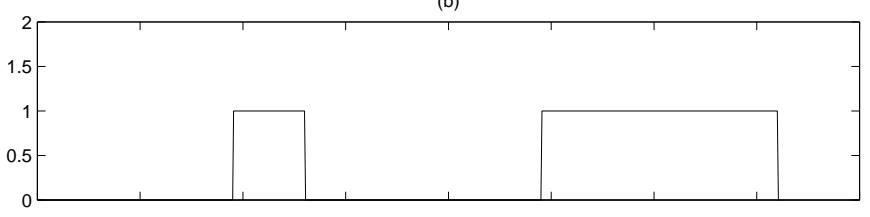

(c)

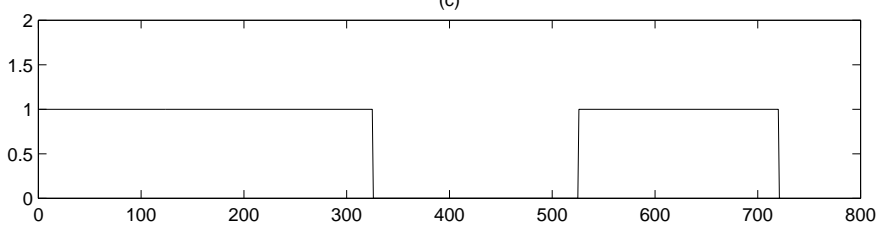

Fig. 3. The detecting results of two different methods for Vastus Lateralis muscle group; (a) the original raw EMG signal, (b) the result of Bonato's method, (c) the result of present method.

analysed to reject spurious transitions (or pulses) whose width are less than 30 samples.

\section{Computation Steps Used for Present Method}

The steps for present method are similar to those of Bonato's, with some differences:

1) instead of load one stride of these EMG signals, $\nu$ strides of EMG signals are loaded, and are time aligned with heel strike synchronization signal

2) the auxilliary sequence $\left\{z_{i}\right\}$ is obtained via Eq.3

3) solving Eq.11 by setting $m=10$ (its time width is the same as $m=5$ in Bonato's methd) $r_{0}=1$ and $P_{f a}=$ 0.05 ; selecting the reasonable root as $P_{\zeta}$

4) substituting the values of $\sigma_{n}$ and $P_{\zeta}$ into Eq. 8 and the first threshold $\zeta$ is obtained

5) following steps are the same as steps 7) to 8) in Bonato's method.

Two muscle activation parameters are frequently used in clinical applications: the Activation Interval defined as activation time of the muscle in one stride normalized with respect stride duration; and On-set time, the time instant when muscle is activated normalized to stride duration. In Table I the parameters of activation interval, on-set time and computation time of this study are shown. The activation interval and onset time are normalized as the percentage of time of a stride (from heel strike to heel strike) and the computation time is in unit of second. The Visual Detection is the average value obtained with visual inspection by four trained observers.

\section{Discussion AND CONCLUSION}

The detection of muscle activation intervals (muscle on or muscle off) provides important clinical information since it allows the investigation of temporal activation pattern of muscle groups, for example during gait. Because of this quite a few methods have been proposed and compared[3], [5]. 
TABLE I

COMPARISON THE RESULTS OF THE TWO DETECTION METHODS WITH THE SAME FALSE-ALARM PROBABILITY

\begin{tabular}{ccccc}
\hline Muscle & Parameters & Bonato Method & Present method & Visual Detection \\
\hline \multirow{2}{*}{ Vastus Lateralis } & Activation Interval & $40.2 \%$ & $69.0 \%$ & $64.7 \pm 4 \%$ \\
& On-set Times & $66.5 \%$ & $71.7 \%$ & $74.3 \pm 2 \%$ \\
& Computation Time & $\sim 17 \mathrm{~s}$ & $<0.1 \mathrm{~s}$ & - \\
\hline \multirow{2}{*}{ Biceps Femoris } & Activation Interval & $49.8 \%$ & $72.0 \%$ & $68.8 \pm 5 \%$ \\
& On-set Time & $72.0 \%$ & $72.7 \%$ & $70.6 \pm 2 \%$ \\
& Computation Time & $\sim 17 \mathrm{~s}$ & $<0.1 \mathrm{~s}$ & - \\
\hline
\end{tabular}

(a)

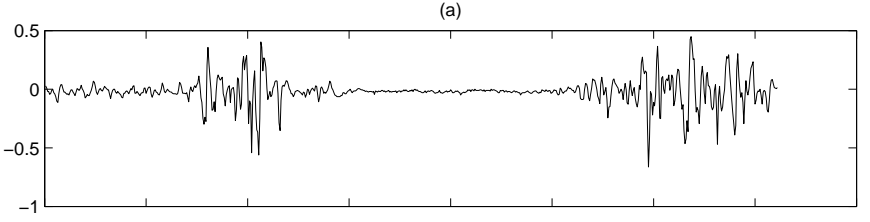

(b)

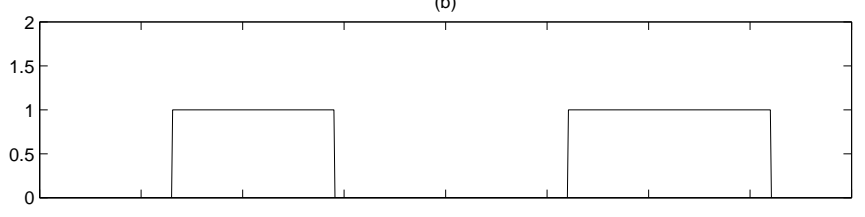

(c)

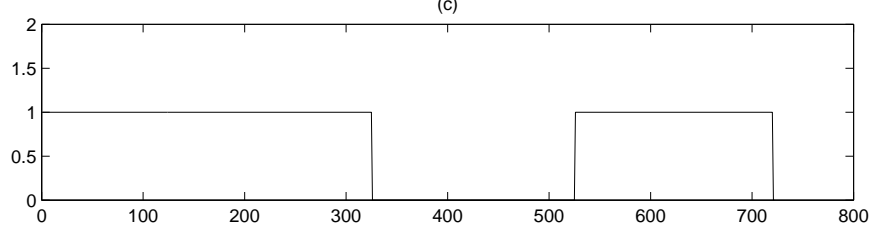

Fig. 4. The detecting results of the two methods for Biceps Femoris muscle group; (a) the original raw EMG signal, (b) the result of Bonato's method, (c) the result of present method.

The challenge is how to perform signal estimation from noise contaminated EMG. Generally, visual inspection is considered to provide highly accurate event detection, because all details of the signal can be assessed by the expertise of expert. For specific applications, besides the accuracy in the detection, the speed of the algorithm can be an important consideration [3]. Algorithms which with high computational time are unsuitable for online detection. One specific drawback to the method of Bonato et al. [2] is that in order for the detection probability $\left(P_{d}\right)$ to be maximum when $P_{f a}$ is fixed, the second threshold has to be chosen as equal to one. This implies that the second threshold is fixed during detection, which implies that the double-threshold detector actually becomes single threshold detector.

The method proposed in this study does not require the signal whitening step which is needed previously. The signal whitening precess takes a lot of computational time as shown in Table I. Moreover the whitening process reduces the signal to noise ratio and consequently reduce the detection probability of the signal, especially when the muscle is activated at low contraction level. This feature will causes the detector to miss a part of activation interval as can be seen in Table I. The detector results (Activation Interval and On-set time) of present method are closer to the visual detection values. One possible disadvantage of the proposed method is the requirement to have multiple samples from repetitions of activity; however, for studies such as gait analysis samples from multiple steps are uniformly available.

The methods proposed in this study provides a fast and more reliable muscle on-off detection. It may be a useful tool for the analysis of surface EMG signal recorded during movement, especially for human kinematics study.

\section{ACKNOWLEDGMENT}

The authors would like to thank Mr. Daniel Theoret in School of Human Kinetics at University of Ottawa for providing the surface EMG data.

\section{REFERENCES}

[1] G. A. Bekey, C. W. Chang, J. Perry, and M. M. Hoffer, "Pattern recoginition of multiple EMG signals applied to the description of human gait", Proc. IEEE, vol. 65, pp. 674-681, 1985.

[2] P. Bonato, T. D'Alessio and M. Knaflitz, "A statistical method for the measurement of muscle activation intervals from surface myoelectric signal during gait", IEEE Trans. Biomed. Eng., vol. 45, pp. 287-299, 1998.

[3] A. Merlo, D. Farina, and R. Merletti, "A fast and reliable technique for muscle activity detection from surface EMG signals", IEEE Trans. Biomed. Eng., vol.50, pp. 316-323, 2003.

[4] A. Papoulis, Probability, Random Variables, and Stochastic Processes. 3rd Edition, New YorK: McGraw-Hill, 1991.

[5] G. Staude and W. Wolf, "Objective motor response onset detection in surface myoelectric signals”, Med. Eng. Phys., vol. 21, pp. 449-467, 1999.

[6] R. G. Shiavi and P. Griffin, "Representing and clustering electromyographic gait patterns with multivariate techniques", Med. Biol. Eng. Comput., vol. 19, pp. 605-611, 1981.

[7] D. A. Winter, "Pathologic gait diagnosis with computer-averaged electromyographical profiles", Arch. Phys. Med. Rehab., vol. 65, pp. 393-398, July 1984 . 